\title{
NMDA EPSCs at Glutamatergic Synapses in the Spinal Cord Dorsal Horn of the Postnatal Rat
}

\author{
Rita Bardoni, ${ }^{1}$ Pier Cosimo Magherini, ${ }^{1}$ and Amy B. MacDermott ${ }^{2}$ \\ ${ }^{1}$ Department of Biomedical Sciences, University of Modena, 41100 Modena, Italy, and ${ }^{2}$ Department of Physiology and \\ Cellular Biophysics and the Center for Neurobiology and Behavior, Columbia University, New York, New York 10032
}

In rat dorsal horn, little is known about the properties of synaptic NMDA receptors during the first two postnatal weeks, a period of intense synaptogenesis. Using transverse spinal cord slices from postnatal day 0-15 rats, we show that $20 \%$ of glutamatergic synapses tested at low-stimulation intensity in spinal cord laminae I and II were mediated exclusively by NMDA receptors. Essentially all of the remaining glutamatergic EPSCs studied were attributable to the activation of both NMDA and AMPA receptors. Synaptic NMDA receptors at pure and mixed synapses showed similar sensitivity to membrane potential, independent of age, indicating similar $\mathrm{Mg}^{2+}$ sensitivity. Kinetic

Glutamate is the principal fast excitatory neurotransmitter in the rat superficial dorsal horn. Synaptically released glutamate activates postsynaptic AMPA and NMDA receptors (Yoshimura and Jessell, 1990; Yoshimura and Nishi, 1993). However, the frequency with which NMDA receptors are present at glutamate synapses in the superficial dorsal horn and their basic kinetic and voltage-dependent properties have not been investigated. Despite this lack of specific information, it is known that NMDA receptors in the dorsal horn are important in the generation and maintenance of several forms of central sensitization associated with allodynia and hyperalgesia (Dickenson et al., 1997). Furthermore, in the adult rat after peripheral nerve injury, NMDA receptors have been shown to be required for reestablishing narrowed receptive fields after peripheral regeneration of afferents (Lewin et al., 1994). Thus, these receptors appear to have multiple roles in the regulation of sensory transmission in the spinal cord dorsal horn, yet little information is available concerning the synaptic currents mediated by NMDA receptors there.

NMDA receptors have a role in spinal cord development. Synaptic NMDA receptor activation may help regulate dendritic outgrowth and establish final synaptic connections in developing rat dorsal horn, as has been suggested for ventral horn neurons (Kalb and Hockfield, 1990; Kalb, 1994). NMDA receptors may influence or mediate the dramatic changes in receptive field size that evolve over the first few postnatal weeks (Fitzgerald, 1985). One form of glutamatergic EPSC that has been proposed to be

\footnotetext{
Received March 27, 1998; revised May 29, 1998; accepted June 2, 1998.

This work was supported by the Whitehall Foundation, the National Science Foundation (A.B.M.), and Ministero Dell'Università e Della Ricerca Scientifica e Tecnologica (R.B. and P.C.M.). We thank Steve Siegelbaum, Gary Westbrook, and Jianguo Gu for helpful comments on an earlier version of this manuscript, and Bob Hawkins, Michael Meyers, and Emilia Bagiella for helpful discussions on data analysis.

Correspondence should be addressed to Rita Bardoni, Department of Biomedical Sciences, Section of Physiology, Via Campi, 287, I-41100, Modena, Italy. E-mail: bardoni@unimo.it

Copyright (ㄷ) 1998 Society for Neuroscience $\quad 0270-6474 / 98 / 186558-10 \$ 05.00 / 0$
}

properties of NMDA EPSCs from pure and mixed synapses were measured at $+50 \mathrm{mV}$. The $10-90 \%$ rise times of the pure NMDA EPSCs were slower (16 vs $10 \mathrm{msec}$ ), and the decay $\tau$ values were faster ( $\tau 1,24$ vs $42 \mathrm{msec} ; \tau 2,267$ vs $357 \mathrm{msec})$ than NMDA EPSCs at mixed synapses. Our results indicate that NMDA receptors are expressed at glutamatergic synapses at a high frequency, either alone or together with AMPA receptors, consistent with the prominent role of NMDA receptors in central sensitization (McMahon et al., 1993).

Key words: NMDA receptor; spinal cord; dorsal horn; development; synaptic transmission; pure NMDA synapse

developmentally important is the pure NMDA EPSC at which only NMDA receptors are present, often called silent synapses (Isaac et al., 1995; Liao et al., 1995; Durand et al., 1996). Some of the earliest evidence for silent or ineffective synapses was obtained by recordings made in the spinal cord (Wall, 1977; Malenka and Nicoll, 1997). Evidence for pure NMDA synapses was first found by Dale and Roberts (1985) in a study on the spinal cord ventral horn of Xenopus embryos. The presence of pure NMDA EPSCs in the developing rat dorsal horn has only recently been reported (Bardoni et al., 1997b; Zhuo and Li, 1997).

We have investigated the basic properties of NMDA receptormediated synaptic currents in the rat superficial dorsal horn during the first two postnatal weeks. NMDA receptors were found to be expressed both alone and together with AMPA receptors, and the kinetics and voltage dependence of NMDA EPSCs were measured. All synaptic NMDA receptors were strongly voltage-dependent. The kinetics of the NMDA receptormediated synaptic currents at synapses with NMDA and AMPA receptors compared with those with NMDA receptors alone were significantly different. No change in synaptic current kinetics was detectable over 2 weeks of postnatal development.

The results of this work have been presented in preliminary form (Bardoni et al., 1996, 1997a,b).

\section{MATERIALS AND METHODS}

Slice preparation. Postnatal day 0-15 ( $\mathrm{P} 0-\mathrm{P} 15)$ rats were anesthetized and decapitated, and the cervical spinal cord was removed and placed in oxygenated ice-cold Krebs' solution. Dorsal and ventral laminectomies were performed, and the spinal cord was isolated and embedded in agarose $\left(2.5 \%\right.$ in Krebs' solution; $\left.36-38^{\circ} \mathrm{C}\right)$. Transverse slices $300-400$ $\mu \mathrm{m}$ thick were cut and then incubated in oxygenated Krebs' solution at $35^{\circ} \mathrm{C}$ for $1 \mathrm{hr}$ and used for recording.

Solutions. Slices were perfused at room temperature with $95 \% \mathrm{O}_{2}-5 \%$ $\mathrm{CO}_{2}$ saturated Krebs' solution (in mM: $125 \mathrm{NaCl}, 2.5 \mathrm{KCl}, 25 \mathrm{NaHCO}_{3}$, $1 \mathrm{NaH}_{2} \mathrm{PO}_{4}, 25$ glucose, $1 \mathrm{MgCl}_{2}$, and $\left.2 \mathrm{CaCl}_{2}, \mathrm{pH} 7.4,320 \mathrm{mOsm}\right)$. Bath solution $\left(0 \mathrm{Mg}^{2+}\right)$ was obtained by omitting $\mathrm{MgCl}_{2}$ from the Krebs' 
solution. Ten micromolar bicuculline and $5 \mu \mathrm{M}$ strychnine were added to the perfusion bath in all experiments to block the strong GABAergic and glycinergic input. The intracellular solution contained (in $\mathrm{mM}$ ): 130 $\mathrm{Cs}^{+}$-gluconate, $10 \mathrm{CsCl}, 11$ EGTA, $1 \mathrm{CaCl}_{2}, 10 \mathrm{HEPES}$, and 2 $\mathrm{Mg}_{2} \mathrm{ATP}, \mathrm{pH}$ adjusted to 7.3 with $\mathrm{NaOH}$, osmolarity adjusted to $305-310$ mOsm with sucrose. The drugs used included (in $\mu \mathrm{M}$ ): 10 6-cyano-7nitroquinoxaline-2,3-dione (CNQX), 10 6-nitro-7-sulfamoyl benzo[F] quinoxaline-2,3-dione (NBQX), $50 \mathrm{D}(-)$-2-amino-5-phosphonopentanoic acid (D-APV) (Tocris Cookson, Bristol, England), 10 bicuculline methiodide, and $5 \mu \mathrm{M}$ strychnine (Sigma, St. Louis, MO). These compounds were superfused in the extracellular solution.

Recording and stimulation. Patch-clamp recordings in whole-cell configuration were obtained from neurons in laminae I and II. The neurons were visually identified using a Zeiss (Oberkochen, Germany) Axioskop microscope fit with a $10 \times$ lens, a $63 \times$ water-immersion lens, and a CCD video camera (Sony, Tokyo, Japan). Lamina II was recognized as a translucent band in the external part of the dorsal horn. Lamina I neurons were identified as the cells localized in the thin region between lamina II and white matter.

The focal stimulation of the tissue around the postsynaptic neuron under study was obtained by moving a glass pipette ( $5 \mu \mathrm{m}$ tip diameter) around the cell in a perimeter of $\sim 100 \mu \mathrm{m}$ from the cell body until a synaptic response was evoked. Stimuli had intensities ranging from 2 to $20 \mathrm{~V}$ (stimulus isolation unit output) and a duration of $0.3 \mathrm{msec}$. Stimulation frequency in most experiments was $0.5 \mathrm{~Hz}$.

The recording electrodes were made from thick-walled borosilicate glass and had a resistance of $\sim 5 \mathrm{M} \Omega$. Whole-cell recordings were obtained in voltage-clamp configuration; usually a $70 \%$ series resistance compensation was introduced. Data were recorded and acquired using an Axopatch-1D amplifier and pClamp 5.5 software (both from Axon Instruments, Foster City, CA). Signals were filtered at $1 \mathrm{kHz}$ and sampled at $2-10 \mathrm{kHz}$. Peak amplitudes and rise time values were measured from averaged EPSCs recorded at $+50 \mathrm{mV}$ and evaluated using pClamp 5.5 software (Clampfit). EPSC decay fits and linear regressions of the data were performed using Sigmaplot software (SPSS, Erkrath, Germany).

Statistical analysis. The data in Figure 2 were expressed as a percentage of the total number of cells (see Fig. $2 A$ ) or synapses (see Fig. $2 B$ ) tested averaged by postnatal day. To test the data for a significant trend over time, we used linear regression. However, the data were required to first be transformed to the corresponding square root values to normalize their distribution (Fleiss, 1986). In Figures 4, 6, and 7, the data are represented with values averaged within 4-day bins. Error bars represent $\mathrm{SD}$ in these figures. To evaluate differences between NMDA receptormediated synaptic currents recorded from the two synaptic types (mixed and pure), as well their age dependency, we used the ANOVA test. Oneand two-way ANOVA was obtained using SPSS software.

Pure NMDA synapse identification procedure. Synapses mediated by NMDA receptors alone, or pure NMDA EPSCs, were identified by recording EPSCs in voltage clamp in the presence of $10 \mu \mathrm{M}$ bicuculline and $5 \mu \mathrm{M}$ strychnine. Initially, each cell was held at $-70 \mathrm{mV}$, and focal stimulation was applied at low frequency $(0.2 \mathrm{~Hz})$. In these conditions, high-intensity focal stimulation (5-20 V) evoked EPSCs mediated mainly by AMPA receptors. To establish the presence of pure NMDA EPSCs, the stimulus intensity was progressively decreased (2-10 V) until AMPA EPSCs disappeared. The membrane was then depolarized to $+50 \mathrm{mV}$, and focal stimuli were applied at the same frequency. The presence of a pure NMDA EPSC was revealed by the appearance of a slow outward EPSC of $10-30 \mathrm{pA}$ amplitude. This minimal stimulation protocol to identify pure NMDA synapses was first used by Liao et al. (1995) and Isaac et al. (1995). Usually five different locations around the postsynaptic cell were tested for the presence of pure NMDA synapses. In a few instances, the increase of the stimulus intensity did not recruit mixed synapses, and only pure NMDA EPSCs were seen.

\section{RESULTS}

\section{Synapses with only NMDA receptors present}

When recording from lamina II neurons that were held under voltage clamp at $-70 \mathrm{mV}$, focal stimulation of the surrounding tissue or primary afferent stimulation evoked fast excitatory synaptic transmission. The evoked EPSCs were mediated predominantly by AMPA receptors with a variable contribution from NMDA receptors (Yoshimura and Jessell, 1990; Bardoni et al., 1996). Because of the voltage dependence of NMDA receptor current flow, however, more systematic tests for synaptic NMDA receptor activation in the presence of $\mathrm{Mg}^{2+}$ were made at positive membrane potentials. To detect pure NMDA EPSCs, stimulation intensity at the focal stimulating electrode was decreased until no fast AMPA receptor-mediated EPSCs were apparent at $-70 \mathrm{mV}$ (Fig. 1A). When the membrane potential was then changed to $+50 \mathrm{mV}$, a slow outward EPSC often became evident. This slow synaptic current at $+50 \mathrm{mV}$ was completely and reversibly blocked by D-APV (Fig. $1 A$ ). Thus, the slow synaptic currents were identified as being mediated by NMDA receptors as defined by the blocking effect of D-APV, effective on all eight neurons tested, and by the strong voltage dependence of the EPSC amplitude. These are referred to here as pure NMDA synapses or pure NMDA EPSCs.

As an additional control to ensure that no AMPA receptors contributed to the pure NMDA synaptic responses, we tested the effect of the AMPA receptor antagonist NBQX on currents considered to be mediated by NMDA receptors alone. Synaptic events were recorded at -70 and $+50 \mathrm{mV}$ in the absence and presence of $10 \mu \mathrm{M}$ NBQX (Fig. $1 B$ ). There was no change in the synaptic current after addition of $\operatorname{NBQX}(n=2)$. To be certain that NBQX was not blocking a small fast AMPA current at +50 $\mathrm{mV}$, the averaged currents with and without NBQX were overlaid, revealing no change in current rise time or peak associated with NBQX application, indicating that no AMPA receptormediated component was present. However, as expected, the synaptic current at $+50 \mathrm{mV}$ was completely blocked by $50 \mu \mathrm{M}$ D-APV.

The frequency of occurrence of pure NMDA synapses was shown to decrease with maturation in several regions of the nervous system (Durand et al., 1996; Wu et al., 1996; Isaac et al., 1997). Therefore, we tested whether the presence of pure NMDA EPSCs was developmentally regulated in lamina II of the spinal cord. As shown in Figure $2 A$, a high percentage (44\%) of lamina II neurons exhibited at least one pure NMDA synapse of the three to five synaptic inputs tested. Figure $2 B$ shows the percentage of pure NMDA synapses over the total number of glutamatergic EPSCs (i.e., pure NMDA receptor-mediated and NMDA plus AMPA receptor-mediated synapses) as a function of age. Averaged over the 2 week test period, $20 \%$ of the total number of synapses tested were mediated by NMDA receptors alone. No significant change in the frequency of neurons expressing pure NMDA synapses or in the relative proportion of pure NMDA synapses was observed in lamina II over the first 2 postnatal weeks.

It is important to note that the percentages of synapses reported in these experiments (Fig. 2) are estimates. Under our stimulation conditions we could only detect pure NMDA EPSCs that had thresholds for stimulation lower than those of mixed receptor EPSCs. In addition, the synaptic responses defined as mixed may actually be attributable to activation of both mixed and pure synapses by the same stimulus intensity (or, less likely, by the activation of pure NMDA and pure AMPA synapses only). From our data, however, we can conclude that a substantial proportion of glutamatergic synapses in lamina II are mediated by NMDA receptors alone.

\section{The amplitude, kinetics, and voltage dependence of pure NMDA EPSCs}

To understand the role of NMDA EPSCs in the transmission of sensory signals in the dorsal horn, it is important to establish the basic parameters of their kinetics and voltage dependence. 
A

CONTROL $-70 \mathrm{mV} \quad$ CONTROL $+50 \mathrm{mV} \quad$ D-APV $+50 \mathrm{mV} \quad$ WASH $+50 \mathrm{mV}$
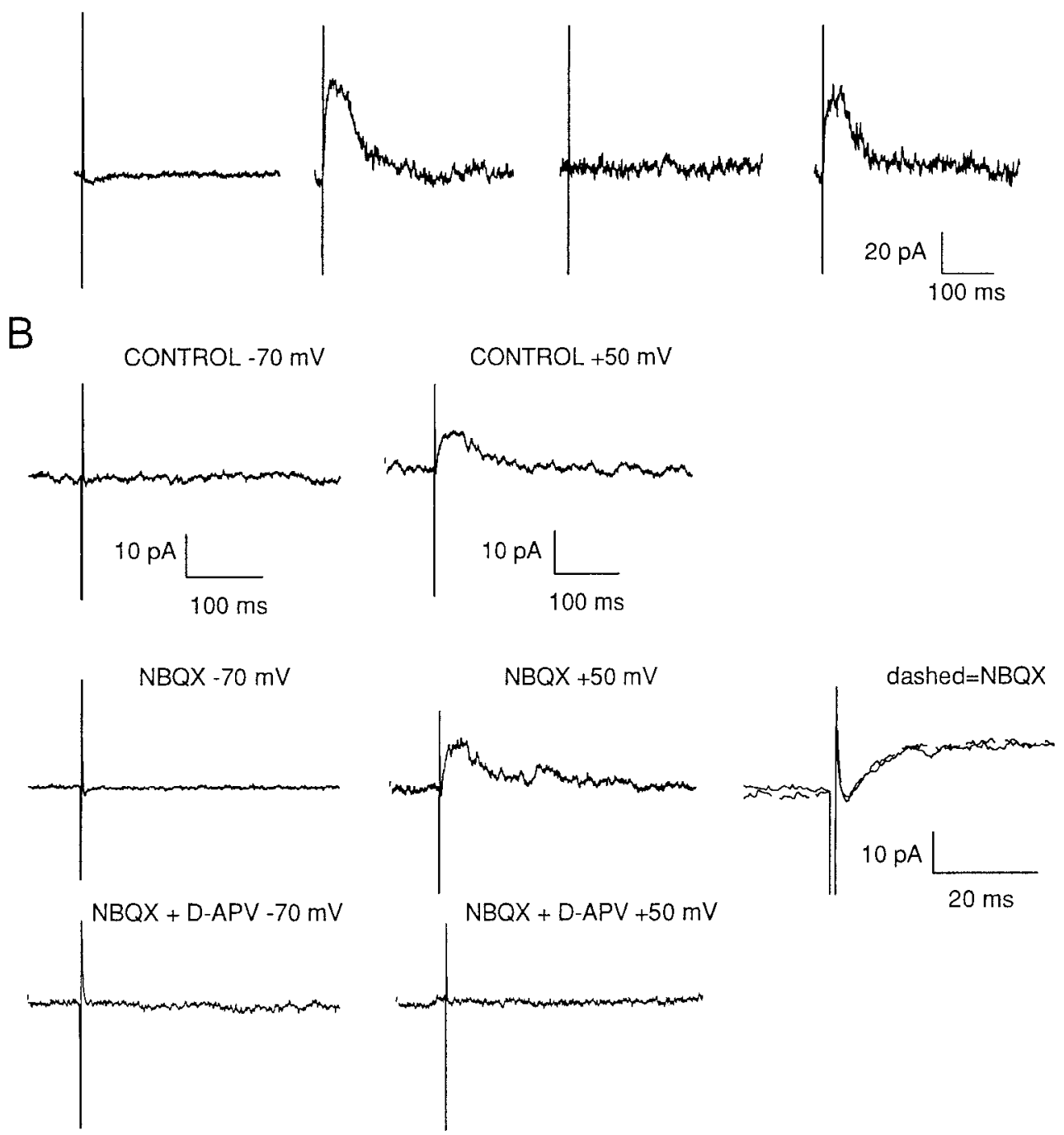

Figure 1. Some glutamatergic synapses evoked in lamina II neurons are mediated by NMDA receptors only. $A$, EPSCs were recorded from a P10 lamina II neuron in $10 \mu \mathrm{M}$ bicuculline and $5 \mu \mathrm{M}$ strychnine at different holding potentials to test for the presence of pure NMDA EPSCs. Stimulation of the neuron held at $-70 \mathrm{mV}$ produced no detectable AMPA EPSC. EPSCs recorded at $+50 \mathrm{mV}$ were completely blocked by $50 \mu \mathrm{M}$ D-APV. After washout of D-APV for $5 \mathrm{~min}$, the NMDA EPSCs tested at $+50 \mathrm{mV}$ recovered. All currents are averages of three traces. $B$, EPSCs were recorded from a P3 lamina II neuron in $10 \mu \mathrm{M}$ bicuculline and $5 \mu \mathrm{M}$ strychnine. Application of $10 \mu \mathrm{M}$ NBQX did not affect the EPSC recorded at $+50 \mathrm{mV}$. When the rising phase of the EPSCs recorded in control (solid line) and in NBQX (dashed line) are superimposed, no effect of NBQX is detectable. Adding D-APV to the NBQX containing extracellular solution blocks the EPSC. All currents are averages of five traces.

These variables will strongly influence the activity dependence of synaptic NMDA receptor activation. Examples of pure NMDA EPSCs recorded from a P1 and a P10 neuron held at $+50 \mathrm{mV}$ are shown in Figure 3. On average, peak amplitudes of pure NMDA EPSCs were small, $26.9 \pm 14.4 \mathrm{pA}(n=17)$, with a slow rise time and current decay time course that was fit by one (data not shown) or two exponentials (Fig. 3). The average rise time of EPSCs at pure NMDA synapses was $15.9 \pm 3.2 \mathrm{msec}(n=14)$. Rise times did not change significantly over the postnatal period under study (see Fig. 7). EPSCs from two neurons were best fit with a singleexponential function giving decay $\tau$ values of 40 and $65 \mathrm{msec}$. EPSCs from 14 cells were best fit with two exponentials with average decay: $\tau 1,24.4 \pm 6.9 \mathrm{msec}$ and $\tau 2,267.2 \pm 95.3 \mathrm{msec}$.
The amplitude of the first component was $15.9 \pm 9.7 \mathrm{pA}$, and the amplitude of the second component was $11.6 \pm 5.7 \mathrm{pA}$.

Pure NMDA EPSCs were strongly suppressed at negative membrane potentials, suggesting potent block by $\mathrm{Mg}^{2+} . I-V$ relationships obtained from three lamina II neurons at different ages (P1, P4, and $\mathrm{P} 13)$ are represented in Figure $4 A$. In all three examples, maximum peak inward current for the pure NMDA EPSC is at approximately $-30 \mathrm{mV}$. Figure $4 B$ shows the ratio of pure NMDA EPSC amplitude recorded at -70 and at $+50 \mathrm{mV}$ as a function of age $(n=22)$. One-way ANOVA of these data indicates no age dependence of the ratio value. Thus, no change in voltage dependence of the synaptic current at pure NMDA synapses occurred over the first 2 weeks of postnatal development, indicating that the $\mathrm{Mg}^{2+}$ sensitivity of NMDA EPSCs at 

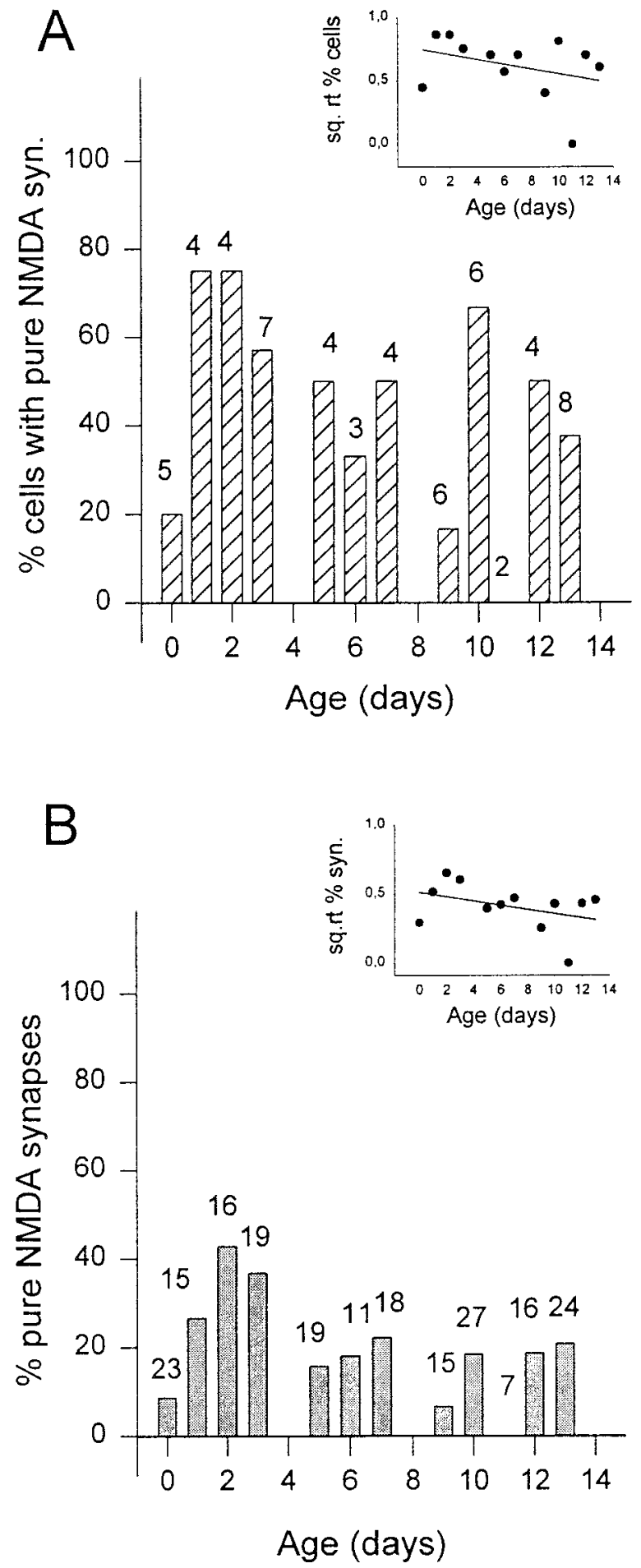

Figure 2. Analysis of the proportion of pure NMDA synapses during the first 2 weeks of postnatal development. $A$, Bars at each postnatal day represent the percentage of lamina II neurons in which pure NMDA EPSCs were observed over the total number of tested cells. The number over each bar is the number of cells tested at that age. Inset, Linear regression fit to the square root of the percentage of cells at each age $(r=$ $0.33 ; p>0.05) . B$, Bars represent the percentage of pure NMDA synapses over the total number of glutamatergic (i.e., NMDA plus AMPA) EPSCs for each age evoked by focally stimulating lamina II neurons. The number over each bar is the number of synapses tested at that age. Inset, Regression fit to the square root of the percentage of synapses at each age $(r=$ $0.39 ; p>0.05)$.
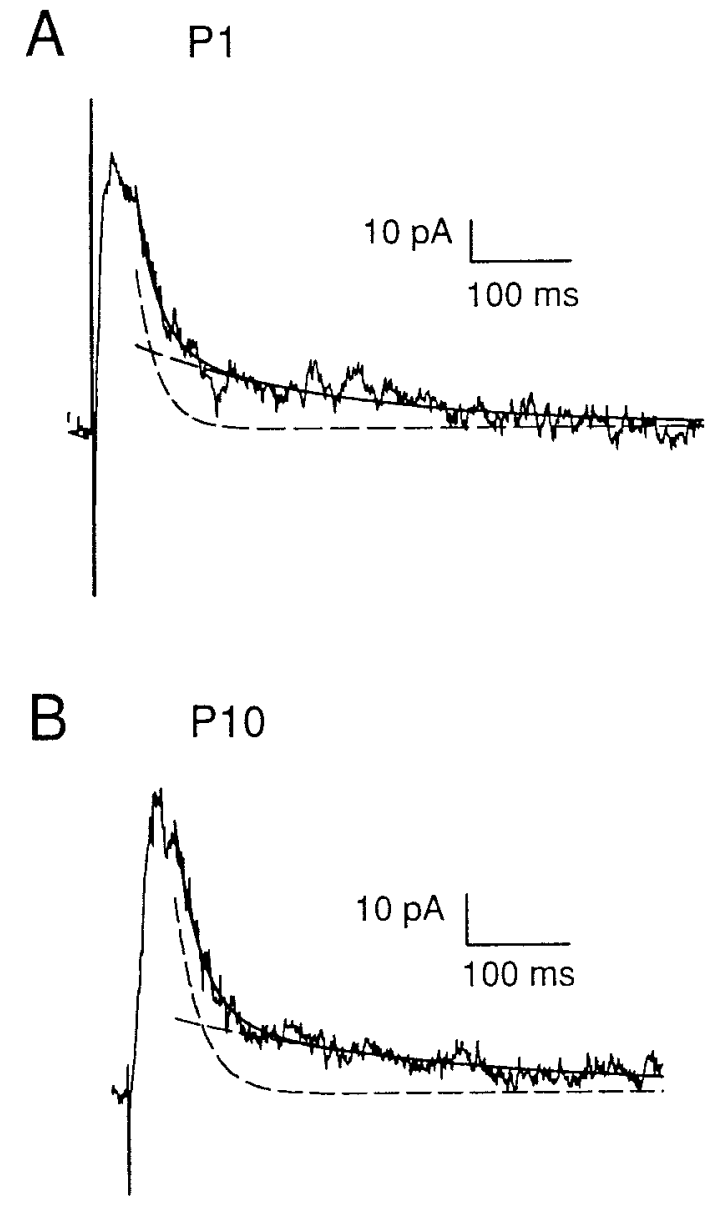

Figure 3. Kinetic analysis of pure NMDA EPSCs. A, EPSCs were recorded from a P1 lamina II neuron held at $+50 \mathrm{mV}$, averaged, and fit with a double-exponential function. The current is the average of five traces. The double-exponential curve is superimposed on the EPSC decay phase (solid line), whereas the two components of the double-exponential function are represented by dashed lines $(\tau 1,21.4 ; \tau 2,209 \mathrm{msec}) . B$, EPSCs were recorded from a P10 lamina II neuron held at $+50 \mathrm{mV}$, averaged, and fit with a double-exponential function $(\tau 1,25.3 ; \tau 2,292 \mathrm{msec})$. The current is the average of five traces.

pure NMDA synapses remains strong throughout postnatal development.

\section{The amplitude, kineticism, and $\mathrm{Mg}^{2+}$ sensitivity of NMDA EPSCs pharmacologically isolated from mixed synapses}

Although substantial proportions of glutamatergic synapses are composed solely of NMDA receptors, other glutamatergic synapses in laminae I and II are mediated by AMPA and NMDA receptors. Focal stimulation was applied at high intensity, evoking synaptic currents that included both AMPA and NMDA components. Under these conditions, the recorded NMDA EPSCs could be attributable to the activation of receptors expressed both at pure NMDA synapses and at mixed synapses (AMPA plus NMDA or AMPA alone). Thus, the synapses contributing to the kinetics of NMDA EPSCs from mixed synapses are more heterogeneous than those of pure NMDA EPSCs. To establish the properties of synaptic NMDA receptors activated under these conditions and to compare them to those expressed at pure NMDA synapses, we determined the voltage dependence and kinetics of the associated synaptic currents. As shown in Figure 5, 
A

P 1

P 4

P 13
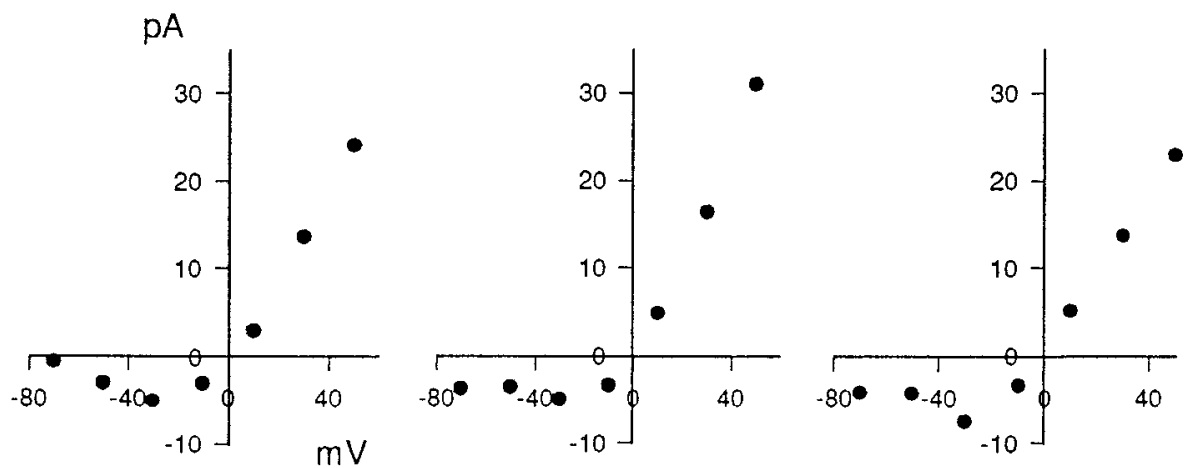

Figure 4. Pure NMDA EPSCs are strongly voltage-dependent. $A, I-V$ relationships obtained by plotting the peak amplitude of averaged pure NMDA EPSCs recorded in bicuculline and strychnine from lamina II neurons as a function of holding potential at different ages $(\mathrm{P} 1, \mathrm{P} 4$, and $\mathrm{P} 13)$. Each point was determined by averaging five EPSC traces. $B$, Voltage dependence of NMDA receptors at pure NMDA synapses was assessed by calculating the ratio of peak synaptic current amplitudes recorded at $-70 \mathrm{mV}$ and +50 $\mathrm{mV}$ in bicuculline and strychnine from lamina II neurons. Average ratio values and SDs are plotted in $4 \mathrm{~d}$ bins and compared as a function of age. One-way ANOVA revealed no relationship between voltage dependence and age $(F=0.91 ; p=$ 0.457).

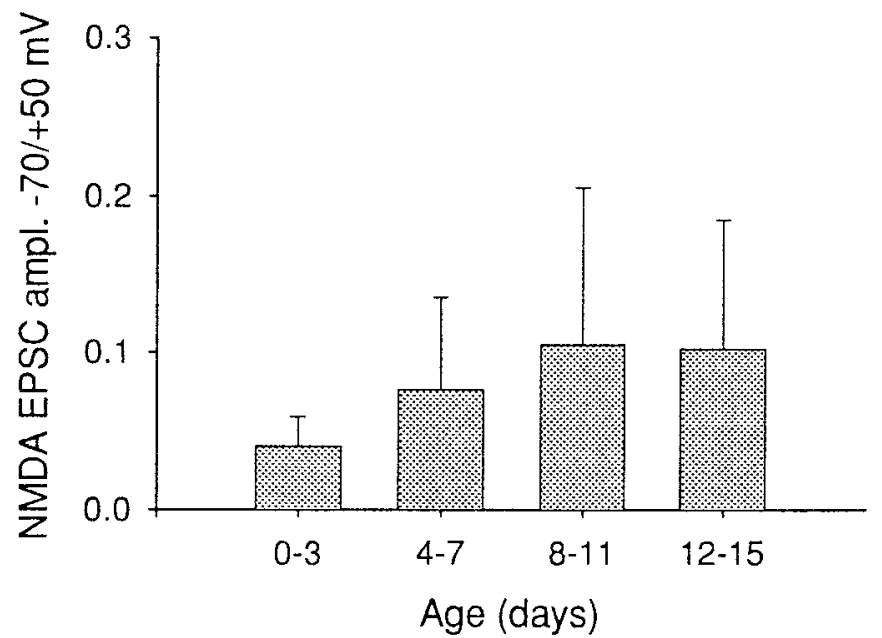

stimulation of a lamina II P4 neuron held at $-70 \mathrm{mV}$ evoked a fast EPSC mediated by AMPA receptors, as indicated by strong block with $10 \mu \mathrm{M}$ CNQX. A small NMDA receptor-mediated component of the EPSC was apparent at $-70 \mathrm{mV}$ in the presence of CNQX (Fig. $5 A$ ) but was much more clearly seen by depolarizing the membrane to $+50 \mathrm{mV}$ (Fig. 5B). Fifty micromolar D-APV completely and reversibly blocked the remaining NMDA EPSC. Nearly all glutamatergic synapses tested in this way revealed evidence of an NMDA component to the EPSC.

Similar to pure NMDA EPSCs, NMDA EPSCs from a mixture of glutamatergic EPSCs were strongly sensitive to membrane potential from birth. Figure $6 A, B$ shows NMDA EPSCs recorded from a lamina II P3 neuron. In the presence of $1 \mathrm{~mm}$ extracellular $\mathrm{Mg}^{2+}$ (Fig. 6A), almost no current was evoked when membrane potential was held at $-70 \mathrm{mV}$, although a large outward EPSC was apparent at $+50 \mathrm{mV}$, indicating strong voltage dependence of these EPSCs. When the bath was switched to one with nominally $0 \mathrm{Mg}^{2+}$, a large EPSC was present at both -70 and $+50 \mathrm{mV}$ (Fig. $6, B)$. By removal of bath $\mathrm{Mg}^{2+}$, the voltage-dependent block of NMDA EPSCs was diminished, as shown by the loss of negative slope conductance (Fig. 6A,B). NMDA receptor $\mathrm{Mg}^{2+}$ sensitivity at mixed glutamatergic synapses was assessed as a function of age

by calculating the ratio of the peak amplitudes of synaptic NMDA currents recorded from lamina I and II neurons at -70 and at $+50 \mathrm{mV}$, as illustrated in Figure $6 C(n=33)$. Similar to pure NMDA EPSCs, the voltage dependence of NMDA receptors at mixed glutamatergic synapses was unchanged throughout the first two postnatal weeks. Furthermore, when the voltage-dependent block of NMDA EPSCs for lamina I neurons was considered separately from lamina II neurons by regression analysis performed on raw data, there was no significant change with age in either group (data not shown). When the ratio of NMDA EPSC amplitudes measured at -70 and $+50 \mathrm{mV}$ was compared between mixed and pure NMDA synapses, they were found to be similar (one-way ANOVA, $p=0.772$ ), indicating that pure and mixed NMDA synapses show comparable $\mathrm{Mg}^{2+}$ sensitivity.

Kinetic properties of NMDA EPSCs at mixed synapses were determined as a function of postnatal age and compared with those of pure NMDA EPSCs. In Figure $7 A$, rise times of NMDA EPSCs isolated from mixed and pure synapses are plotted as a function of age. Mean rise time measured at mixed synapses for both lamina I and II neurons was $10.5 \pm 2.7 \mathrm{msec}(n=23)$. As was the case for the pure NMDA EPSCs, there was no change in rise time detected over the first two postnatal weeks. However, 


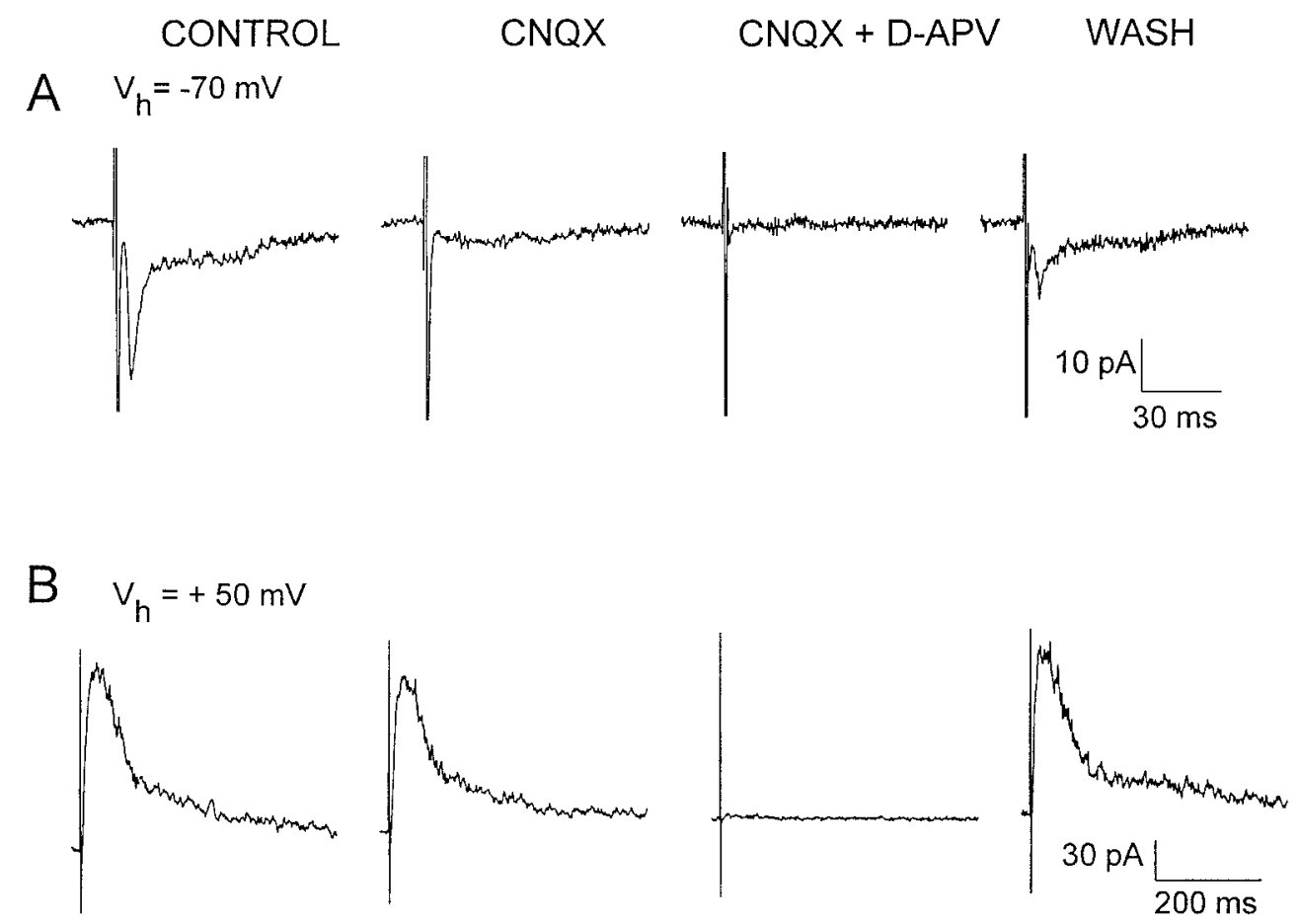

Figure 5. NMDA EPSCs isolated from mixed glutamatergic synapses in laminae I and II over the first 2 postnatal weeks. $A$, EPSCs were recorded from a P4 lamina II neuron held at $-70 \mathrm{mV}$ and assessed for sensitivity to different antagonists. Glutamatergic EPSCs evoked in bicuculline and strychnine are mediated by AMPA receptors, blocked by $10 \mu \mathrm{M}$ CNQX and NMDA receptors, blocked by $50 \mu \mathrm{M}$ D-APV. The antagonist effects were reversible after a 5 min wash. $B$, EPSCs were recorded from the same neuron under the same conditions as in $A$, except the membrane potential was held at +50 $\mathrm{mV}$. All currents are the average of five traces.

rise times of mixed NMDA EPSCs were significantly faster than those of pure NMDA EPSCs (two-way ANOVA, $p \ll 0.01$ ). The significant difference in rise times between the two types of NMDA EPSCs indicates that some aspect of the synapses, such as molecular composition of the NMDA receptors or source of the transmitter, must be different (see Discussion).

The decay phase of NMDA EPSCs at mixed synapses was best fit by a double-exponential function in 23 of 24 neurons from laminae I and II recorded at $+50 \mathrm{mV}$. The decay $\tau$ for the one cell with a single-exponential fit was $58 \mathrm{msec}$. Figure $7 B, C$ shows the values of $\tau 1$ and $\tau 2$ as a function of age $(\tau 1,41.9 \pm 11.25 \mathrm{msec} ; \tau 2$, $357.14 \pm 132.96 \mathrm{msec} ; n=23$ ) for the mixed synapse NMDA EPSCs. The corresponding amplitudes of the two components are $32.0 \pm 23.6$ and $18.4 \pm 13.4 \mathrm{pA}$. The $\tau$ values obtained for NMDA EPSCs at mixed synapses were compared with those determined for pure NMDA EPSCs that also required fitting with a double-exponential function. $\tau 1$ and $\tau 2$ values of mixed and pure NMDA EPSCs are plotted in Figure $7 B, C$. Using a two-way ANOVA, decay $\tau$ remained constant over time for both pure or mixed synapses. However, there was a significant difference between mixed and pure NMDA EPSC groups for $\tau 1(p \ll 0.01)$, whereas no significant difference was detected for $\tau 2$. The differences in rise times and decay $\tau 1$ both suggest a fundamental difference in the NMDA EPSCs at the two different types of synapses.

\section{DISCUSSION}

We have investigated the properties of synaptic NMDA receptors of the rat spinal cord dorsal horn during postnatal development and found that postsynaptic NMDA receptors contribute to synaptic transmission at many glutamatergic synapses in laminae I and II by birth. At some synapses, the NMDA receptors are the only glutamate receptors present (Bardoni et al., 1997a,b; Zhuo and $\mathrm{Li}, 1997)$, whereas other synapses are likely to include combinations of AMPA and NMDA receptors.

\section{The kinetics of NMDA EPSCs in the superficial dorsal horn}

All of the NMDA EPSCs recorded from neurons in laminae I and II decayed with fast kinetics at $+50 \mathrm{mV}$. The average decay $\tau$ values measured at pure NMDA synapses were 24 and 267 msec, whereas the average decay $\tau$ values at mixed synapses were 42 and $357 \mathrm{msec}$. Decay kinetics of NMDA EPSCs have been shown to be determined by channel kinetics of the NMDA receptor (Lester et al., 1990; Rosenmund et al., 1995) that in turn are influenced by the subunits that contribute to receptor composition. Initially, the NR2D subunit appeared to be the major NR2 subunit expressed in spinal cord at embryonic day 17 (Monyer et al., 1994) and in mature dorsal horn neurons (Tölle et al., 1993). However, single-channel recordings from dorsal horn neurons have shown that channels with different conductance levels are present with proposed subunit composition, including NR2A/B (higher conductance) and NR2D (lower conductance) (Momiyama et al., 1996). Most recently, in situ hybridization and single-cell PCR studies in dorsal horn have reported that the most common subunit composition of NMDA receptor at the cervical level is NR1, NR2A, B and D, with NR2A predominant over B and D (Karlsson et al., 1997; Sjödin et al., 1997). The rapid kinetics we have reported for dorsal horn neuron NMDA EPSCs suggest a strong influence of the NR2A subunit on receptor configuration, because receptors expressed in heterologous ex- 
(1)

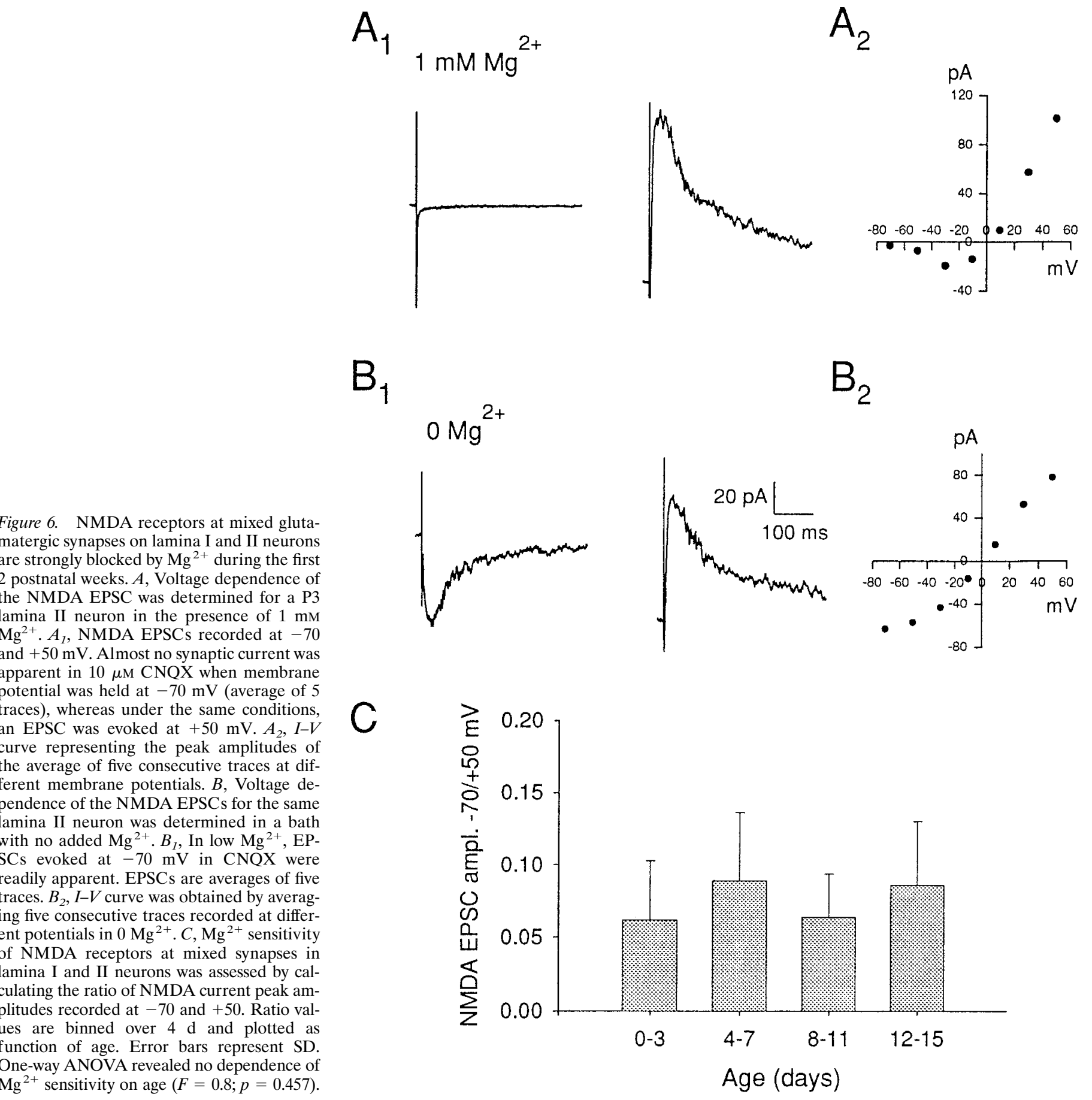

Figure 6. NMDA receptors at mixed glutamatergic synapses on lamina $I$ and II neurons lamina II neuron in the presence of $1 \mathrm{~mm}$ $\mathrm{Mg}^{2+} . A_{1}$, NMDA EPSCs recorded at -70 and $+50 \mathrm{mV}$. Almost no synaptic current was apparent in $10 \mu \mathrm{M}$ CNOX when membrane potential was held at $-70 \mathrm{mV}$ (average of 5 traces), whereas under the same conditions, EPSC was evoked at $+50 \mathrm{mV} . A, I-V$ lamina II neuron was determined in a bath with no added $\mathrm{Mg}^{2+} . B_{1}$, In low $\mathrm{Mg}^{2+}$, EPSCs evoked at $-70 \mathrm{mV}$ in CNQX were readily apparent. EPSCs are averages of five traces. $B_{2}, I-V$ curve was obtained by averaging five consecutive traces recorded at differA nessed by cal$\mathrm{Mg}^{2+}$ sensitivity on age $(F=0.8 ; p=0.457)$.

pression systems (Monyer et al., 1994) and in developing neurons (Flint et al., 1997) that include NR2A have rapid kinetics.

Developmental changes in NMDA EPSC kinetics have been observed in several regions of the CNS (Carmignoto and Vicini, 1992; Hestrin, 1992; Ramoa and McCormick, 1994; Khazipov et al., 1995; Shi et al., 1997). In these studies, the decay kinetics became faster as development progressed. The most common pattern has been that the decay $\tau$ of the NMDA EPSC is fit by a single-exponential function at younger ages, whereas a doubleexponential function is required at older ages. Khazipov et al. (1995) has proposed that the contribution of the fast component increases with age. In dorsal horn neurons, fits of the NMDA
EPSC decay required two exponentials at all postnatal ages, and decay $\tau$ values are comparable to the values obtained from NMDA EPSCs in mature systems (Carmignoto and Vicini, 1992; Khazipov et al., 1995). These observations further support the idea that the kinetics of the NMDA EPSCs in superficial dorsal horn, and possibly the associated subunit composition of the synaptic NMDA receptors, are in a relatively mature configuration by birth.

\section{Possible explanations for differences in NMDA EPSC} kinetics recorded as pure and mixed synapses

Kinetic analysis of pure NMDA EPSCs evoked in dorsal horn neurons has revealed that these currents have slower rise times 
A

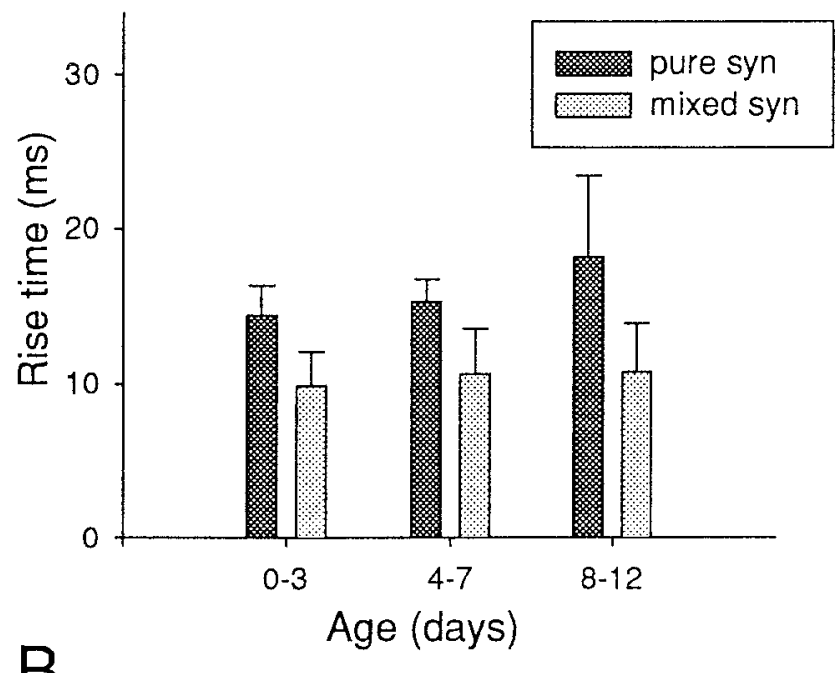

B
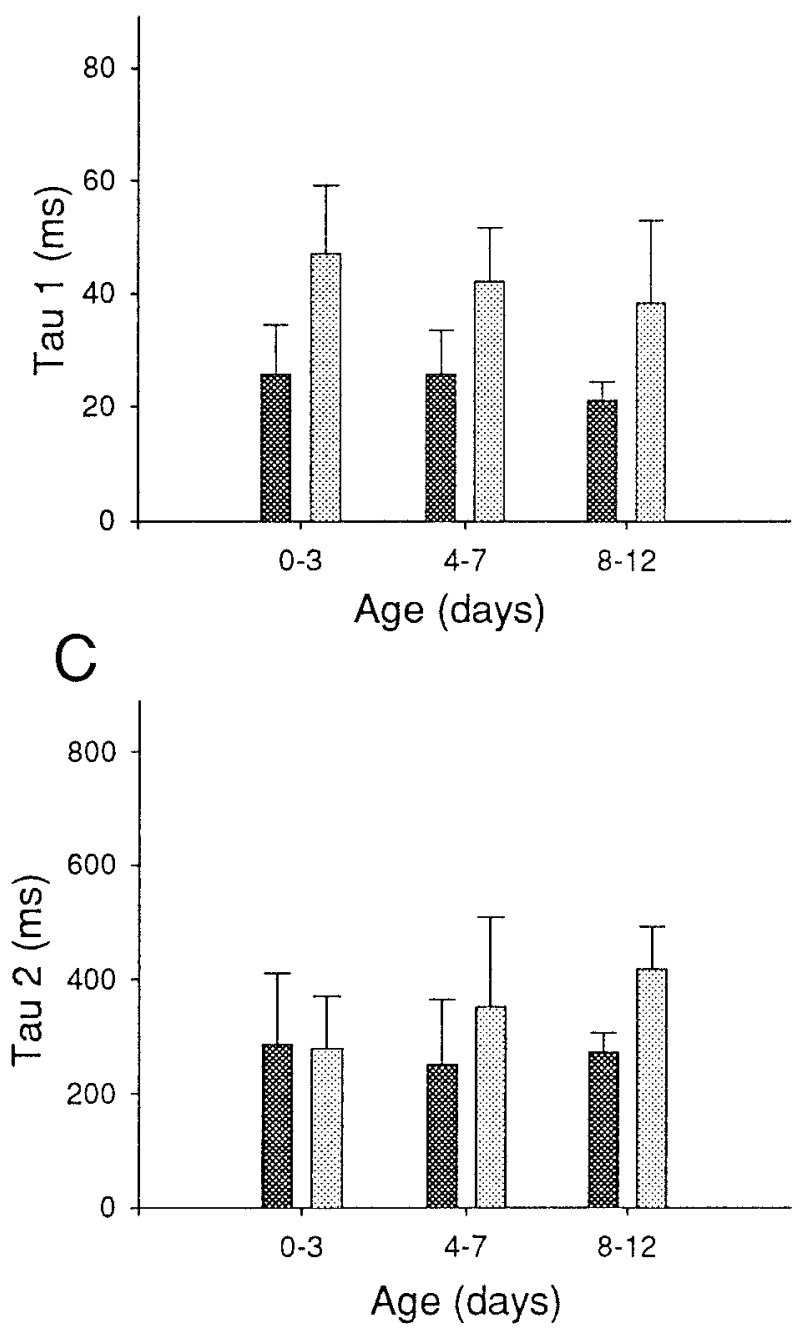

Figure 7. Kinetic analysis of NMDA EPSCs at mixed synapses and comparison with pure NMDA EPSCs. $A$, Ten to $90 \%$ rise time values determined from NMDA EPSCs at mixed and pure NMDA synapses are binned and plotted as a function of age. The first two bins are $4 \mathrm{~d}$, and the third bin includes data from $5 \mathrm{~d}$. Two-way ANOVA indicates no dependence of rise time on age for either type of synapse $(F=1.43 ; p=0.254)$ and a highly significant difference in rise time of pure and mixed NMDA and faster decay $\tau 1$ s than NMDA EPSCs at mixed synapses do. There are several possible explanations for the observed differences in EPSC kinetics.

If we make the assumption that rise time and decay $\tau$ values of NMDA EPSCs are principally determined by channel kinetics (Lester et al., 1990; Rosenmund et al., 1995) and that channel kinetics are mainly determined by receptor subunit composition, then it is NMDA receptor subunit composition that favors faster kinetics at pure synapses. NR2A subunit has been shown to display fast kinetics (Monyer et al., 1994), and NR2A expression correlates highly with fast NMDA EPSC decay $\tau$ values in developing neocortical neurons (Flint et al., 1997). Vicini et al. (1998) have recently shown that NMDA receptors expressed in HEK 293 cells composed of NR1 and NR2A subunits have deactivation kinetics similar to the decay $\tau$ values we report for pure NMDA EPSCs. Specifically, they found that after $1 \mathrm{msec}$ application of 1 mM Glu, currents decayed with a fast $\tau$ of $33 \mathrm{msec}$ and a slow $\tau$ of $247 \mathrm{msec}$ (compared with 24 and $267 \mathrm{msec}$ for pure NMDA EPSCs, respectively). When cells were cotransfected with NR1, $\mathrm{NR} 2 \mathrm{~A}$, and NR2B, the fast and slow decay $\tau$ values became proportionally slower as the relative amount of NR2B was increased. For example, when NR1, NR2A, and NR2B receptor subunit cDNAs were added in 1:3:1 proportions, average decay $\tau$ values were 43 and $387 \mathrm{msec}$. This compares favorably with the 42 and 357 msec decay $\tau$ values measured from NMDA EPSCs from mixed synapses. These data provide a good molecular hypothesis for the NMDA EPSC decay kinetics reported in our studies. The hypothesis is that the decay $\tau$ values of the pure NMDA EPSCs are attributable to receptors composed only of NR1 and NR2A subunits. NMDA receptors at other synapses would include additional NR2 subunits, such as NR2B.

Alternatively, the slower rise time of the pure synapses could indicate that they are located at electrotonically distant sites from the recording electrode and that the slow rise time is simply attributable to the low-pass filtering of the dendritic membrane (Rall and Segev, 1991). Because the decay $\tau$ values are faster at pure NMDA synapses, dendritic filtering cannot account for the difference in decay time. It has been suggested that the decay $\tau$ values of NMDA EPSCs are voltage-dependent (Hestrin, 1992). Thus, it is possible to account for faster decay $\tau$ values at these putatively remote pure NMDA synapses because of the inability of the voltage clamp to bring the membrane potential at the synapse to $+50 \mathrm{mV}$. These EPSCs would decay at a faster rate than synapses closer to the soma because of the more negative membrane potential at the site of channel opening. It is also possible that EPSC decay $\tau$ would be faster if remote synapses were preferentially subject to shunting by activation of other channels, although both $\mathrm{GABA}_{\mathrm{A}}$ and glycine receptors were blocked in our experiments.

A third explanation for why pure NMDA EPSCs have a slower

$\leftarrow$

synapses $(F=27.57 ; p \ll 0.01) . B$, The decay phase of NMDA EPSCs at mixed and pure synapses was fit with a double-exponential function. $\tau 1$ values are plotted in bins as a function of age. The decay $\tau 1$ values of the two types of NMDA EPSCs are significantly different (two-way ANOVA, $F=25.44 ; p \ll 0.01$ ), although neither changes significantly as a function of age $(F=1.032 ; p=0.37)$. $C, \tau 2$ values determined from NMDA EPSCs at mixed and pure synapses are binned and plotted against age. $\tau 2$ values for mixed and pure synapses are not significantly different (two-way ANOVA, $F=3.4 ; p=0.074)$ nor are they dependent from age $(F=0.661$; $p=0.524)$. All kinetic parameters were determined from averaged EPSCs (5-15 traces) recorded at $+50 \mathrm{mV}$. Error bars indicate SD. 
time to peak than mixed EPSCs is that pure NMDA EPSCs are attributable to spillover of synaptically released glutamate onto receptors on neighboring neurons, either at synaptic sites (Kullmann et al., 1996) or even extrasynaptic sites. If this is true, the diffusion of glutamate to receptors mediating pure NMDA responses could become rate-limiting for the EPSC rise time rather than channel kinetics, thus explaining the slow rise time observed in our studies. A diffusion-limited rise time would not, however, predict the faster decay $\tau$ we have observed at pure NMDA synapses. In this case, the faster decay $\tau 1$ would have to be explained by some other mechanism such as one of those outlined above.

\section{Role of NMDA EPSCs in the spinal cord dorsal horn}

Evidence for an important role for NMDA receptors in nociceptive processing in dorsal horn is becoming increasingly strong. Glutamate is released from both primary afferents and at least some of the local interneuronal connections (Willis and Coggeshall, 1991). The synaptic activation of NMDA receptors appears to contribute to both the induction and maintenance of many forms of central sensitization associated with hyperalgesia and allodynia (Dickenson et al., 1997). However, little is known about which synaptic connections express postsynaptic NMDA receptors. Our data suggest that postsynaptic NMDA receptors are ubiquitous throughout the superficial dorsal horn. They were detected on every neuron tested in our study and activated under a variety of stimulation conditions. Nearly all of the NMDA EPSCs decayed with both fast and slow $\tau$ values, on the order of tens and several hundreds of milliseconds, respectively. With pain-evoked action potentials coming in to dorsal horn neurons at $5-20 \mathrm{~Hz}$ (Bessou and Perl, 1969), these slow EPSPs driven by NMDA EPSCs are expected to show strong summation. Furthermore, because of the strong voltage dependence of the NMDA synaptic currents in the rat dorsal horn (Figs. 4, 6), this summation is expected to be highly nonlinear and to powerfully influence the output firing of the dorsal horn neurons. Finally, tetanic orthodromic activation of some dorsal horn neurons initiates an NMDA receptor-dependent sequence of events resulting in long-term changes in synaptic transmission referred to as longterm potentiation (LTP) and long-term depression (LTD) (Randic et al., 1993; Sandkuhler et al., 1997). The voltage dependence of current flow through the NMDA receptor is believed to provide the sensitivity to specific tetanic activity in hippocampal NMDA receptor-dependent LTP and LTD. We have shown that by birth, synaptic NMDA receptors throughout the dorsal horn express a strong voltage-dependent $\mathrm{Mg}^{2+}$ block, endowing them with the ability to function as activity detectors in the dorsal horn.

\section{REFERENCES}

Bardoni R, Magherini PC, MacDermott AB (1996) Magnesium sensitivity of synaptic NMDA receptors in rat spinal cord lamina I and II neurons during postnatal development. Soc Neurosci Abstr 22:797.

Bardoni R, Magherini PC, MacDermott AB (1997a) NMDA receptor mediated synaptic transmission in rat spinal cord lamina I and II during postnatal development. Pflügers Arch 434:R59.

Bardoni R, Magherini PC, MacDermott AB (1997b) Some glutamatergic synapses on neurons in rat spinal cord lamina II are mediated by NMDA receptors alone. Soc Neurosci Abstr 23:2284.

Bessou P, Perl ER (1969) Response of cutaneous sensory units with unmyelinated fibers to noxious stimuli. J Neurophysiol 32:1025-1043.

Carmignoto G, Vicini S (1992) Activity-dependent decrease in NMDA receptor responses during development of the visual cortex. Science 258:1007-1011.

Dale N, Roberts A (1985) Dual-component amino-acid-mediated synaptic potentials: excitatory drive for swimming in Xenopus embryos. J Physiol (Lond) 363:35-59.

Dickenson AH, Chapman V, Green GM (1997) The pharmacology of excitatory and inhibitory amino acid-mediated events in the transmission and modulation of pain in the spinal cord. Gen Pharmacol 38:633-638.

Durand GM, Kovalchuk Y, Konnerth A (1996) Long-term potentiation and functional synapse induction in developing hippocampus. Nature 381:71-75.

Fitzgerald M (1985) The post-natal development of cutaneous afferent fibre input and receptive field organization in the rat dorsal horn. J Physiol (Lond) 364:1-18.

Fleiss JL (1986) The design and analysis of clinical experiments. New York: Wiley.

Flint AC, Maisch US, Weishaupt JH, Kriegstein AR, Monyer H (1997) NR2A subunit expression shortens NMDA receptor synaptic currents in developing neocortex. J Neurosci 17:2469-2476.

Hestrin S (1992) Developmental regulation of NMDA receptormediated synaptic currents at a central synapse. Nature 357:686-689.

Isaac JTR, Nicoll RA, Malenka RC (1995) Evidence for silent synapses: implications for the expression of LTP. Neuron 15:427-434.

Isaac JTR, Crair MC, Nicoll RA, Malenka RC (1997) Silent synapses during development of thalamocortical inputs. Neuron 18:269-280.

Kalb RG (1994) Regulation of motor neuron dendrite growth by NMDA receptor activation. Development 120:3063-3071.

Kalb RG, Hockfield S (1990) Induction of a neuronal proteoglycan by the NMDA receptor in the developing spinal cord. Science 250:294-296.

Karlsson H, Carlqvist H, Sjödin J, Eriksson A, Brandin L, Wikström L, Wallin J, Näsström J (1997) Functional characteristics of NMDA receptor mediated whole-cell currents and NMDA receptor mRNA expression in single superficial dorsal horn neurons. Soc Neurosci Abstr 23:930.

Khazipov R, Ragozzino D, Bregestovski P (1995) Kinetics and $\mathrm{Mg}^{2+}$ block of $N$-methyl-D-aspartate receptor channels during postnatal development of hippocampal CA3 pyramidal neurons. Neuroscience 69:1057-1065.

Kullmann DM, Erdemli G, Asztely F (1996) LTP of AMPA and NMDA receptor-mediated signals: evidence for presynaptic expression and extrasynaptic spill-over. Neuron 17:461-474.

Lester RAJ, Clements JD, Westbrook GL, Jahr CE (1990) Channel kinetics determine the time course of NMDA receptor-mediated synaptic currents. Nature 346:565-567.

Lewin GR, Mckintosh W, McMahon SB (1994) NMDA receptors and activity-dependent tuning of the receptive fields of spinal cord neurons. Nature 369:482-485.

Liao D, Hessler NA, Malinow R (1995) Activation of postsynaptically silent synapses during pairing-induced LTP in CA1 region of hippocampal slice. Nature 375:400-404.

Malenka RC, Nicoll RA (1997) Silent synapses speak up. Neuron 19:473-476.

McMahon SB, Lewin GR, Wall PD (1993) Central hyperexcitability triggered by noxious inputs. Curr Opin Neurobiol 3:602-610.

Momiyama A, Feldmeyer D, Cull-Candy SG (1996) Identification of a native low conductance NMDA channel with reduced sensitivity to $\mathrm{Mg}^{2+}$ in rat central neurones. J Physiol (Lond) 494:479-492.

Monyer H, Burnashev N, Laurie DJ, Sakmann B, Seeburg PH (1994) Developmental and regional expression in the rat brain and functional properties of four NMDA receptors. Neuron 12:529-540.

Rall W, Segev I (1991) Space-clamp problems when voltage clamping branched neurons with intracellular microelectrodes. In: Voltage and patch clamping with microelectrodes (Smith Jr TG, Lecar H, Redman SJ, Gage PW, eds), pp 191-215. Baltimore: Waverly.

Ramoa AS, McCormick DA (1994) Enhanced activation of NMDA receptor responses at the immature retinogeniculate synapse. J Neurosci 14:2098-2105.

Randic M, Jiang MC, Cerne R (1993) Long-term potentiation and longterm depression of primary afferent neurotransmission in the rat spinal cord. J Neurosci 12:5228-5241.

Rosenmund C, Feltz A, Westbrook GL (1995) Synaptic NMDA receptor channels have a low open probability. J Neurosci 15:2788-2795. 
Sandkuhler J, Chen JG, Cheng G, Randic M (1997) Low-frequency stimulation of afferent Adelta-fibers induces long-term depression at primary afferent synapses with substantia gelatinosa neurons in the rat. J Neurosci 17:6483-6491.

Shi J, Aamodt SM, Constantine-Paton M (1997) Temporal correlations between functional and molecular changes in NMDA receptors and GABA neurotransmission in the superior colliculus. J Neurosci 17:6264-6276.

Sjödin J, Fernandes H, Karlsson U, Näsström J, Enkvist K, Wallin L, Wikström L (1997) NMDA receptor subunit expression in the spinal cord. Soc Neurosci Abstr 23:930.

Tölle TR, Berthele A, Zieglgänsberger W, Seeburg PH, Wisden W (1993) The differential expression of 16 NMDA and non-NMDA receptor subunits in the rat spinal cord and in periacqueductal gray. J Neurosci 13:5009-5028.

Vicini S, Wang JF, Li JH, Zhu WJ, Wang YH, Luo JH, Wolfe BB, Grayson DR (1998) Functional and pharmacological differences be tween recombinant $N$-methyl-D-aspartate receptors. J Neurophysiol 79:555-566.

Wall PD (1977) The presence of ineffective synapses and the circumstances which unmask them. Philos Trans R Soc Lond B Biol Sci 278:361-372.

Willis WD, Coggeshall RE (1991) Sensory mechanisms of the spinal cord. New York: Plenum.

Wu G-Y, Malinow R, Cline HT (1996) Maturation of a central glutamatergic synapse. Science 274:972-976.

Yoshimura M, Jessel TM (1990) Amino acid-mediated EPSPs at primary afferent synapses with substantia gelatinosa neurones in the rat spinal cord. J Physiol (Lond) 430:315-335.

Yoshimura M, Nishi S (1993) Blind patch-clamp recordings from substantia gelatinosa neurons in adult rat spinal cord slice: pharmacological properties of synaptic currents. Neuroscience 53:519-526.

Zhuo M, Li P (1997) Synaptic mechanisms contributing to serotonininduced facilitation of nociception in dorsal horn neurons of spinal cord. Soc Neurosci Abstr 23:445. 